\title{
MOBILE PANORAMIC MAPPING USING CCD-LINE CAMERA AND LASER SCANNER WITH INTEGRATED POSITION AND ORIENTATION SYSTEM
}

\author{
R. Reulke ${ }^{\mathrm{a}, *}, \mathrm{~A} . \mathrm{Wehr}^{\mathrm{b}}$ \\ ${ }^{a}$ Institute for Photogrammetry, University of Stuttgart - Ralf.Reulke@ipho,uni-stuttgart.de \\ ${ }^{\mathrm{b}}$ Institute for Navigation, University of Stuttgart - wehr@nav.uni-stuttgart.de
}

KEY WORDS: Digital panoramic camera, laser scanner, data fusion, mobile mapping

\begin{abstract}
:
The fusion of panoramic camera data with laser scanner data is a new approach and allows the combination of high-resolution image and depth data. Different applications are in the field of city modelling and computer vision application, as well as the documentation of the cultural heritage.

Panoramic geometry will be realized by a CCD-line, which is precisely rotated around the projection centre. In the case of other possible movements, the actual position of the projection centre and the view direction has to be measured. Linear moving panoramas e.g. along a wall are an interesting extension of such rotational panoramas. The instantaneous position and orientation determination can be realized with an inertial navigation system.

This paper investigates the combination of a panoramic camera and a laser scanner with a navigation system for indoor and outdoor applications.
\end{abstract}

\section{INTRODUCTION}

Generation of city models offering a high realistic impression requires three-dimensional imaging sensors with high 3D resolution and high image quality.

The idea and the vision of the system, which is described here, is an integrated measurement system for acquiring high resolution image and depth data from extended object, e.g. building facades.

High resolution images can be acquired by CCD-matrix and line sensors. Main advantage of line sensors is the generation of high resolution images without merging or stitching of images patches like in matrix imaging. A problem is an additional sensor motion of the CCD-line to achieve the second image dimension. An obvious solution is the accurate rotation around CCD-line axis on a turn table as used in panoramic imaging.

The depth image or the surfaces of the imaged area can be acquired very precisely in a reasonable short time with laser scanners. However, these systems very often sample only depth data with poor horizontal resolution. Some of them offer monochrome intensity images of poor quality in the spectral range of the laser beam (e.g. NIR). Very few commercial laser scanners use additional imaging colour sensors for obtaining coloured 3D images.

However, with regard to building surveying and setting up cultural heritage archives the imaging resolution of laser scanner data must be improved. This can be achieved by combining data from a high-resolution digital $360^{\circ}$ panoramic camera with data from a laser scanner.

Fusing these image data with 3D information of laser scanning surveys very precise 3D models with detailed texture information will be obtained. This approach is related to $360^{\circ}$ geometry.

Large linear structures like city facades can be acquired with panoramic approach only from different standpoints with variable resolution of the object. To overcome this problem, laser-scanner and panoramic camera should be linear moved along e.g. a building facade.
Applying this technique the main problem is georeferencing and fusing the two data sets of the panoramic camera and the laser scanner respectively. An additional accurate attitude measurement is necessary for each measured CCD- and laserline.

The experimental results shown in the following will deal with this problem and will lead to an optimum surveying setup comprising a panoramic camera, laser scanner, a position and orientation system. This surveying and documentation system will be called POSLAS-PANCAM (POS supported laser scanner panoramic camera).

The vision of this approach is a compact and handy system for combined data acquisition and inspection.

To verify this approach first experiments were carried out with the Digital $360^{\circ}$ Panoramic camera (M1), the 3D-Laserscanner (3D-LS) and a Position and Orientation System (POS).

\section{SYSTEM}

The measurement system consists of a combination of an imaging system, a laser scanner and a system for attitude determination.

\subsection{Digital 360 ${ }^{\circ}$ Panoramic Camera (M2)}

The digital panoramic camera EYESCAN will be preliminary used as a measurement system to create high-resolution $360^{\circ}$ panoramic images for photogrammetry and computer vision (Scheibe, 2001, Klette, 2001). The sensor principle is based on a CCD line, which is mounted on a turntable parallel to the rotation direction. Moving the turntable generates the second image direction. To reach highest resolution and a large field of view a CCD-line with more then 10,000 pixels is used. This CCD is a RGB triplet and allows acquiring true colour images. A high SNR electronic design allows a short capture time for a $360^{\circ}$ scan.

EYESCAN is designed for rugged everyday field use as well as for the laboratory measurement. Combined with a robust and

\footnotetext{
* Corresponding author
} 
powerful portable PC it becomes easy to capture seamless digital panoramic pictures. The sensor system consists of the camera head, the optical part (optics, depth dependencies) and the high precision turntable with DC-gear-system motor.

\begin{tabular}{|l|l|}
\hline Number of Pixel & $3 * 10200$ (RGB) \\
\hline $\begin{array}{l}\text { Radiometric } \\
\text { dynamic/resolution }\end{array}$ & 14 bit / 8 bit per channel \\
\hline Shutter speed & $4 \mathrm{~ms}$ up to infinite \\
\hline Data rate & 15 Mbytes / s \\
\hline $\begin{array}{l}\text { Data volume } 360^{\circ} \quad \text { (optics } \\
\text { f=60mm) }\end{array}$ & 3 GBytes \\
\hline Acquisition time & 4 min \\
\hline Power supply & $12 \mathrm{~V}$ \\
\hline
\end{tabular}

Table 1. Technical parameter of the digital panoramic camera

Table 1 summarise the principle features of the camera: The camera head is connected to the PC with a bidirectional fibre link for data transmission and camera control. The camera head is mounted on a tilt unit for vertical tilt of $\pm 30^{\circ}$ with $15^{\circ}$ stops. Axis of tilt and rotation are in the needlepoint.

The preprocessing of the data consists of data correction (PRNU, DSNU, offsets) and a (non linear) radiometric normalisation to cast the data from 16 to 8 bit. All this procedures can be run in real time or off line. Additional software parts are responsible for real-time visualisation of image data, a fast preview for scene selection and a quick look during data recording.

\subsection{The Laser Scanner 3D-LS}

In the experiments M2 images was supported by the 3D-LS depth data. This imaging laser scanner carries out the depth measurement by side-tone ranging (Wehr, 1999). This means, the optical signal emitted from a semiconductor laser is modulated by high frequency signals. As the laser emits light continuously such laser system are called continuous wave (cw) laser system. The phase difference between the transmitted and received signal is proportional to the two-way slant range. Using high modulation frequencies, e.g. $314 \mathrm{MHz}$, resolutions down to the tenth of a millimetre are possible.

\begin{tabular}{|l|l|}
\hline Laser power & $0.5 \mathrm{~mW}$ \\
\hline Optical wavelength & $670 \mathrm{~nm}$ \\
\hline Inst. field of view (IFOV) & $0.1^{\circ}$ \\
\hline Field of view (FOV) & $30^{\circ} \times 30^{\circ}$ \\
\hline Scanning pattern & $\begin{array}{l}-2 \text {-dimensional line (standard) } \\
- \text { vertical line scan } \\
\text { - free programmable pattern }\end{array}$ \\
\hline Pixels per image & max. $32768 \times 32768$ pixels \\
\hline Range & $<10 \mathrm{~m}$ \\
\hline Ranging accuracy & $\begin{array}{l}0.1 \mathrm{~mm} \text { (for diffuse reflecting } \\
\text { targets, } \rho=60 \%, 1 \text { m distance) }\end{array}$ \\
\hline Measurement rate & $\begin{array}{l}2 \mathrm{kHz} \text { (using on side tone) } \\
600 \mathrm{~Hz} \text { (using two side tones) }\end{array}$ \\
\hline
\end{tabular}

Table 2. Technical parameter of 3D-LS

Besides depth information these scanners sample for each measurement point the backscattered laser light with a 13 bit resolution. Therefore, the user obtains 3D surface images. The functioning of the laser scanner is explained in (Wehr, 1999). Figure 2 shows 3D-LS. The technical parameter are compiled in Table 2

\subsection{Applanix POS-AV 510}

The attitude measurement is the key problem of this combined approach. For demonstration we use the airborne attitude measurement system POS AV 510 from Applanix, which is designed for those applications that require both excellent absolute accuracy and relative accuracy. An example of this would a high altitude, high resolution digital line scanner.

The absolute measurement accuracy after post processing is 5$30 \mathrm{~cm}$ in position, $\delta \theta=\delta \phi=0.005^{\circ}$ for pitch or roll and $\delta \psi=0.008^{\circ}$ for heading.

For an object distance D the angle dependent spatial accuracy d is therefore

$$
d=D \cdot \delta \quad(\delta \text { in } \mathrm{rad})
$$

For an object distance $\mathrm{D}=10 \mathrm{~m}$ the spatial accuracy is $\mathrm{d} \cong 1 \mathrm{~mm}$ and appropriate for verification of a mobile mapping application.

For a future mobile mapping system a simpler attitude measurement, which is also less expensive is necessary. For this purpose we expect in the next few years new gyro development and improved post processing algorithms.

\section{FUSION OF M1 AND 3D-LS DATA}

To investigate the fusion of panoramic and laser data first experiments where carry out in a lab.

\subsection{Experimental Set-up}

In order to study the problems arising by fusion of data sets of the panoramic camera and the 3D-LS, both instruments took an image of a special prepared scene, which were covered with well-defined control points and some 3D objects in a laboratory. The panoramic camera was mounted on a tripod (like in. Figure 1). After recording completion the camera were dismounted and 3D-LS were mounted on the tripod without changing the tripod's position. 3D-LS were used in the imaging mode scanning a field of view (FOV) of $30^{\circ} \times 30^{\circ}$ comprising $800 \times 800$ pixels. Each pixel is described by the quadruple Cartesian coordinates plus intensity (x, y, z, I). The M2 image covers a FOV of approximately $30^{\circ} \times 60^{\circ}$ with $5000 \times 10000$ pixels.

31 control points are available in a distance of $8 \mathrm{~m}$ to $9 \mathrm{~m}$. Lateral resolution of laser and panoramic scanner is $13 \mathrm{~mm}$ and $1.05 \mathrm{~mm}$ respectively, which is a suitable value for fusion of the data sets.

For the coordinate determination of the signalised points an independent approach was done. Using image data from a DCS 460 camera and bundle block adjustment program Australis.

The lateral accuracy of about 30 points is $0.5 \mathrm{~mm}$ and depth accuracy about $3 \mathrm{~mm}$.

\subsection{Modeling and Calibration}

Laser scanner and panoramic camera work with different coordinate systems and must be adjust one to each other. The laser scanner delivers Cartesian coordinates; where as M2 puts out data in a typical photo image projection. Although, both devices were mounted on the same tripod one had to regard that the projection centre of both instruments were not located exactly in the same position. This section describes the geometrical relation between the digital surface data of the laser scanner and the image data from the camera, or the relation between object points and position of image pixel. This task 
needs a model of panoramic imaging and a calibration with known target data.

The imaging geometry of the panoramic camera is characterized by the rotating CCD-line, assembled perpendicular to the $x-y$ plane and forming an image by rotation around the z-axis. The modelling and calibration of panoramic cameras was investigated and published recently (Schneider, 2002 \& 2003, Klette, 2001 \& 2003).

For camera description and calibration we use the following approach (see Figure 3). The CCD-line is placed in the focal plate perpendicular to the $z^{\prime}$-axis and shifted with respect to the $y^{\prime}-z^{\prime}$ coordinate origin by $\left(y_{0}^{\prime}, z_{0}^{\prime}\right)$. The focal plate is mounted in the camera at a distance $\mathrm{x}^{\prime}$, which is suitable to the object geometry. If the object is far from the camera the CCD is placed in the focal plan of the optics at $\mathrm{x}^{\prime}=\mathrm{c}$ (the focal length) on the $\mathrm{x}^{\prime}-$ axis behind the optics (lower left coordinate system). To form an image, the camera is rotated around the origin of a $(\mathrm{x}, \mathrm{y})$ coordinate system.

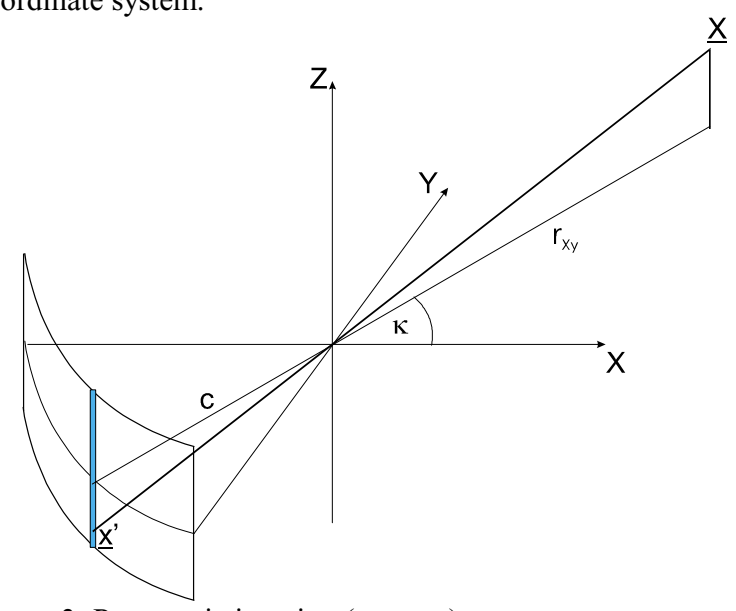

Figure 3: Panoramic imaging (see text)

To derive the relation between object point $\underline{X}$ and a pixel in an image the colinearity equation can be applied.

$$
\underline{\mathrm{X}}-\underline{\mathrm{X}}_{0}=\lambda \cdot\left(\underline{\mathrm{x}}-\underline{\mathrm{x}}_{0}\right)
$$

$\underline{x}$ is the image coordinate, $\underline{X}_{0}$ and $\underline{x}_{0}$ are the projection centre for the object and the image space. To see this object point with a pixel of the CCD-line on the focal plate, the camera has to be rotated by an angle of $\kappa$ around $\mathrm{z}$-axis. For the simplest case $\left(\mathrm{y}_{0}=0\right)$ the result is

$\left(\underline{\mathrm{X}}-\underline{\mathrm{X}}_{0}\right)=\lambda \cdot \underline{\underline{\mathrm{R}}}^{\mathrm{T}} \cdot\left(\underline{\underline{x}^{\prime}}-\underline{\underline{x}}_{0}^{\prime}\right)=\lambda \cdot\left[\begin{array}{ccc}\cos \kappa & -\sin \kappa & 0 \\ \sin \kappa & \cos \kappa & 0 \\ 0 & 0 & 1\end{array}\right] \cdot\left[\begin{array}{c}-\mathrm{c} \\ 0 \\ z^{\prime}-\mathrm{z}_{0}^{\prime}\end{array}\right]=\lambda \cdot\left[\begin{array}{c}-\mathrm{c} \cdot \cos \kappa \\ -\mathrm{c} \cdot \sin \kappa \\ z^{\prime}-\mathrm{z}_{0}^{\prime}\end{array}\right]$

To derive some important parameters of the camera, a simplified approach is used. The unknown scale factor can be calculated from the square of the $x-y$ components of this equation:

$$
\lambda=\frac{\mathrm{r}_{\mathrm{XY}}}{\mathrm{c}} \quad \mathrm{r}_{\mathrm{XY}}=\sqrt{\left(\mathrm{X}-\mathrm{X}_{0}\right)^{2}+\left(\mathrm{Y}-\mathrm{Y}_{0}\right)^{2}}
$$

The meaning of $r_{X Y}$ can easily see in Figure 3. This result is a consequence of the rotational symmetry. By dividing the first two equations and using the scale factor for the third, the following equations deliver an obvious result, which can be geometrically derived from Figure 3 .

$$
\frac{\Delta \mathrm{Y}}{\Delta \mathrm{X}}=\tan \kappa \text { and } \Delta \mathrm{Z}=\mathrm{r}_{\mathrm{XY}} \cdot \frac{\Delta \mathrm{z}^{\prime}}{\mathrm{c}}
$$

The image or pixel coordinates $(i, j)$ are related to the angle $\kappa$ and the $z$-value. Because of the limited image field for this investigation, only linear effects (with respect to the rotation and image distortions) should be taken into account:

$$
\mathrm{i}=\frac{1}{\delta \kappa} \cdot \mathrm{a} \tan \frac{\Delta \mathrm{Y}}{\Delta \mathrm{X}}+\mathrm{i}_{0} \quad \mathrm{j}=\frac{\mathrm{c}}{\delta \mathrm{z}} \cdot \frac{\Delta \mathrm{Z}}{\mathrm{r}_{\mathrm{XY}}}+\mathrm{j}_{0}
$$

$\delta z$

$\delta \mathrm{k} \quad$ angle of one rotation step

c focal length

The unknown or not exactly known parameters $\delta \kappa, \mathrm{i}_{0}$, c and $\mathrm{j}_{0}$ can be derived from known marks in the image field.

For calibration we used signalized points randomly distributed and in different distances from the camera. The analyzing of the resulting errors in the object space shows, that the approach (4) and (5) must be extended. Following effects should be incorporated:

$$
\begin{array}{ll}
- & \text { Rotation of the CCD (around } \mathrm{x} \text {-axis) } \\
- & \text { Tilt of the camera (rotation around y-axis)) }
\end{array}
$$

This effect can be incorporated into equation (2). The variation of the angel $\varphi$ and $\omega$ should be small $(\sin \varphi=j, \cos \varphi=1$ and $\sin \omega=\omega, \cos \omega=1)$

$$
\left(\underline{x}^{\prime}-\underline{x}_{0}^{\prime}\right)=\lambda^{-1} \cdot \underline{\underline{R}} \cdot\left(\underline{X}-\underline{X}_{0}\right)=\lambda \cdot\left[\begin{array}{ccc}
\cos \kappa & \sin \kappa & \omega \cdot \sin \kappa-\varphi \cdot \cos \kappa \\
-\sin \kappa & \cos \kappa & \omega \cdot \sin \kappa+\varphi \cdot \sin \kappa \\
\varphi & -\omega & 1
\end{array}\right] \cdot\left[\begin{array}{c}
X-X_{0} \\
Y-Y_{0} \\
Z-Z_{0}
\end{array}\right]
$$

For this special application the projection centre of the camera is $\left(X_{0}, Y_{0}, Z_{0}\right) \cong(0,0,0)$. With a spatial resection approach, based on equation (6), the unknown parameter of exterior orientation can be derived.

Accuracy of panorama camera model is $\sigma \approx 3$ image pixel of the camera. Using an improved model an accuracy of better then one pixel can be achieved. (Schneider, 2003)

\subsection{Fusion of Panoramic and Laser Scanner Data}

The calibration procedure, as shown in the last section delivers a relation between image coordinates $(i, j)$ and object points $(\mathrm{X}, \mathrm{Y}, \mathrm{Z})$. The classical orthophoto approach however is in the opposite direction. After defining the region, which should be orthorectified, the procedure is the following:

- Discretisation of this region with a defined object pixel distance

- For each location take the height and calculate for the object coordinates $(X, Y, Z)$ the equivalent image point $(\mathrm{i}, \mathrm{j})$

- Put the grey or color value in the object raster

A resulting problem of panoramic geometry is the implicit relation between the pixel coordinates $i$ and the rotation angle $\chi$, which is only solvable for the simplest case (5). Because of CCD-line rotation and camera tilt the pixel coordinate can be determine only in an iterative way. An additional problem results from the small object distance to height relation, which is addressed by the true ortho-rectification. Occludes areas from larger objects will be filled with wrong object texture and some objects seems to be twice. 

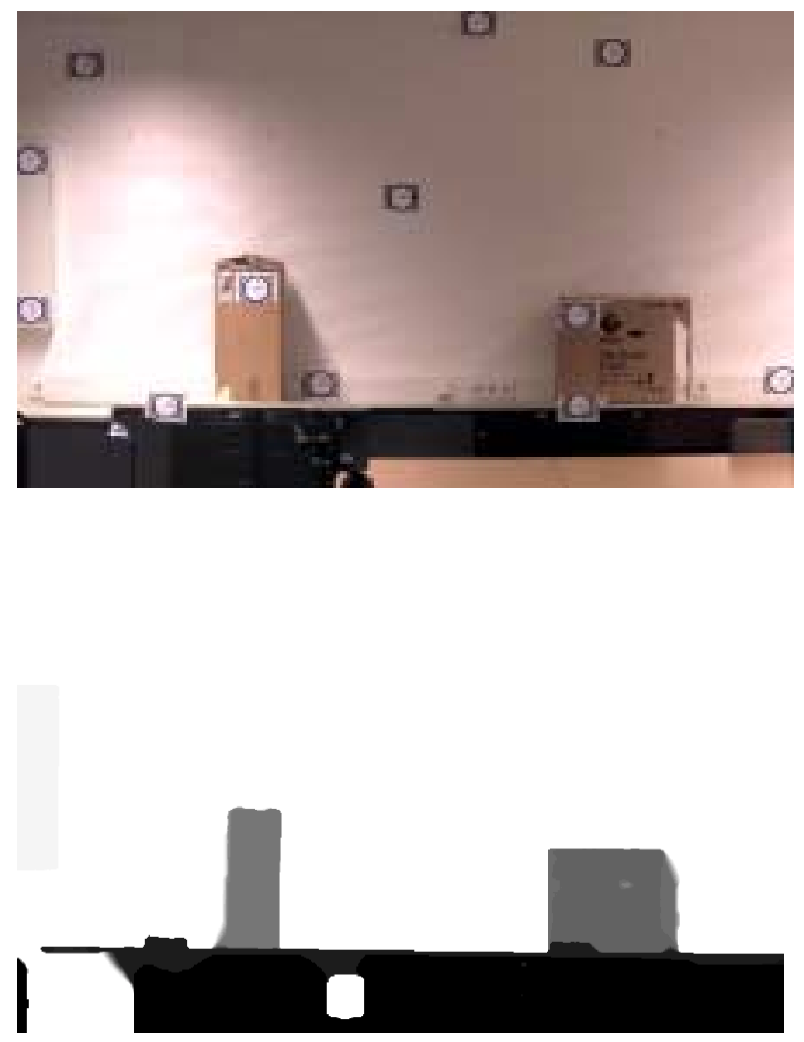

Figure 4: Orthorectified panoramic image (upper image) based on the digital elevation model from laser scanner (lower image)

In the rectified image (upper image) image errors can be observed in the box occluded regions. The white background of the DEM image represents the wall in the background of the left image.

\section{POSLAS-PANCAM}

The results of the preceding experiments leads to an integrated mechanical setup of the M2, 3D-LS and the Applanix POS which is called POSLAS-PANCAM (PLP-CAM). Figure 5 depicts that the centre of rotation of the inertial measurement unit (IMU) of POS, the origin of 3D-LS and the phase centre of the GPS antenna are aligned to a vertical line, which is the axis of rotation. M2 is mounted off axis.

This construction allows a precise relation between 3D-LS and panoramic data and is the main requirement for data fusion. The 3D-LS data are related to the POS data as the lever arms were minimised and are well defined by the construction. The algorithms for transforming the position and orientation data with regard to 3D-LS centre which is defined in the centre of the first scanning mirror (s. Figure 7) are available in the Applanix software POSPROC. For fusing the M2 data with the 3D-data the parallax between M2 and 3D-LS must be taken into account. In a first approximation it can be derived from the construction drawings. However, for precise surveys a special calibration is required which gives the parameters for correcting manufacturing tolerances.

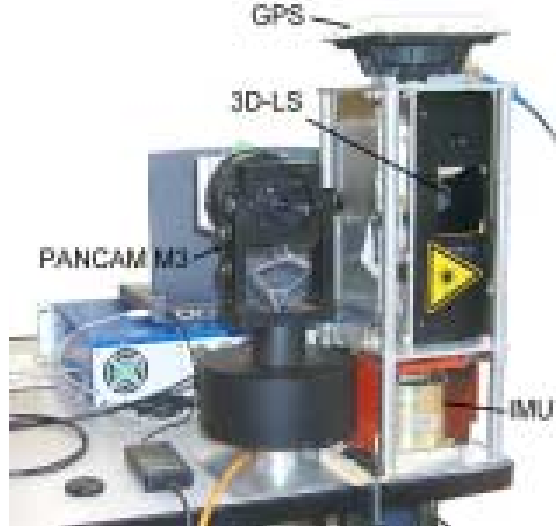

Figure 5: PLP-CAM

For PLP-CAM one main problem is the exact synchronization between the different instruments, because each of it works independently. The block chart in Figure 6 shows the approach. Using event markers solves the problem by generating time stamps. These markers are stored by POS and combine a measurement event with absolute GPS-time (e.g. starting a scanning line).

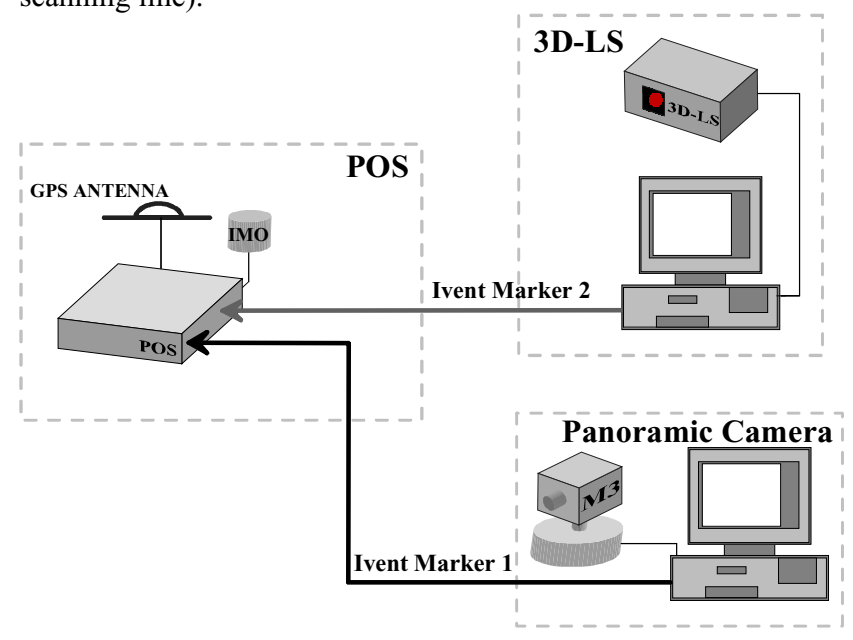

Figure 6: Synchronization

\subsection{Rectification of Line Scanner Data}

First experiments were done with line scanner data from a (nonrotating) moving platform. For rectification we use attitude data from the Applanix system. The rectification was done on a plane and is equivalent to airborne data rectification (Scheele et al. 2001).

Figure 7 shows the original data from the scanned area from a photograph and Figure 8 depicts the original scanned data. 


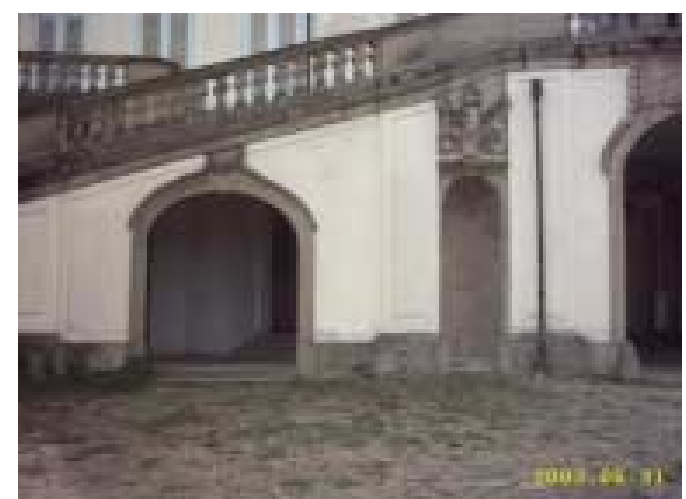

Figure 7 Scanned object (photography)

The result after correction is imaged in Figure 9. The analysis of the attitude data shows, that the amplitude of the disturbing movements of the platform are much more larger than for the airborne application.

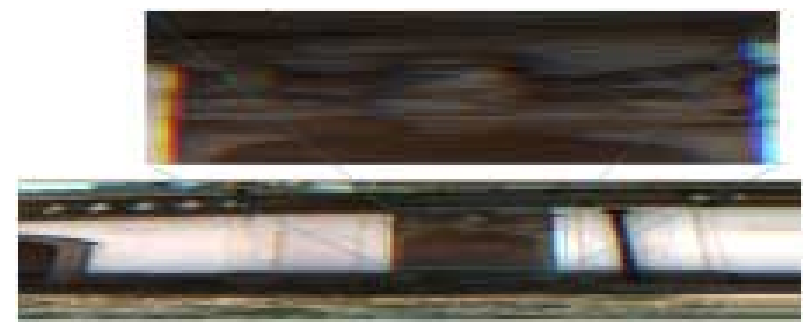

Figure 8: Original scanned data with panoramic camera

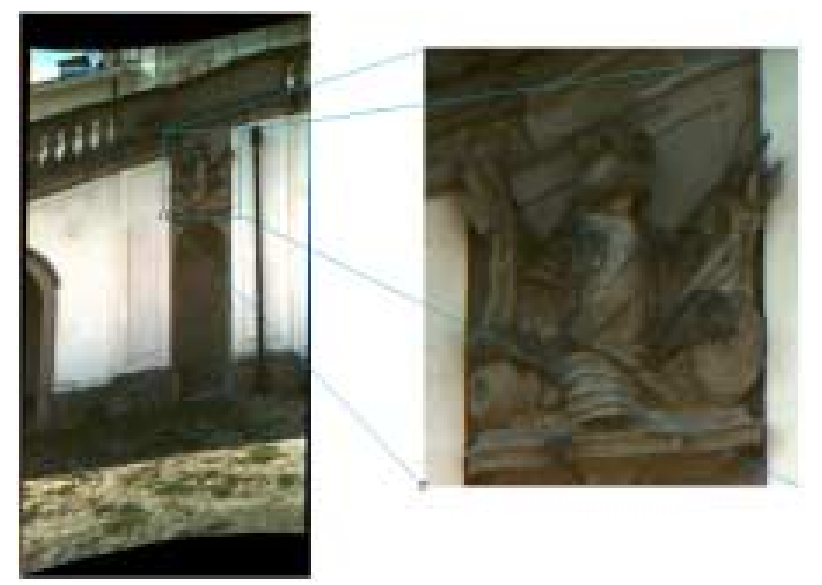

Figure 9: Result after correction

\section{CONCLUSIONS}

The laboratory experiments fusing M3 data with 3D-LS data show that using such an integrated system high resolved 3Dimages can be computed. The processing of the two independent data sets makes clear that a well defined and robust assembly is required. Such an assembly benefits from the well defined locations of the different origins and the relative orientation of the different devices with respect to each other. These parameters must be derived by using a special calibration test field. The experiments with PLP-CAM demonstrated that in courtyards and in narrow street with high buildings one has to face poor GPS signals. Here the POS-AV system of Applanix company was used. It worked very degraded, because it is designed for airborne applications, where one do not have to regard obscurations and multipath effects. For this application POS-LV system of Applanix will deliver improved results.

\section{REFERENCES}

Klette, R.; Gimel'farb, G.; Reulke, R., 2001. Wide-Angle Image Acquisition, Analysis and Visualization, Vision Interface 2001, Proceedings, pp. 114-125.

Klette, R.; Gimelfarb, G.; Huang, F.; Wei, S. K.;Scheele, M.; Scheibe, K.; Börner, A.; Reulke, R. 2003. A Review on Research and Applications of Cylindrical Panoramas, CITRTR-123

Reulke, R.; Scheele, M.; Scheibe, K. u.a., 2001. Multi-SensorAnsätze in der Nahbereichsphotogrammetrie, 21. Jahrestagung DGPF, Konstanz, Sept. 2001, DGPF, Photogrammetrie und Fernerkundung.

Scheele, M., Börner, A., Reulke, R., Scheibe; K., 2001. Geometrische Korrekturen: Vom Flugzeugscanner zur Nahbereichskamera; Photogrammetrie, Fernerkundung, Geoinformation.

Scheibe, K., Korsitzky, H., Reulke, R., Scheele, M., Solbrig, M., 2001. EYESCAN - A high resolution digital panoramic camera, LNCS 1998, Springer, Berlin 2001, pp. 77.

Schneider, D., Maas, H.-G., Geometrische Modellierung und Kalibrierung einer hochauflösenden digitalen Rotationszeilenkamera, DGPF-Tagung, 2002

Schneider, D., 2003. Geometrische Modellierung und Kalibrierung einer hochauflösenden digitalen Rotationszeilenkamera, 2. Oldenburger 3D-Tage, 27.2.

Wehr, A., 1999. 3D-Imaging Laser Scanner for Close Range Metrology. Proc. of SPIE, Orlando, Florida, 6-9 April 1999, Vol. 3707: pp. 381-389.

Australis home page

http://www.sli.unimelb.edu.au/australis/index.htm 